\title{
ON TWO-SIDED ARTINIAN QUOTIENT RINGS
}

\author{
by P. F. SMITH
}

(Received 3 December, 1970)

Djabali [1] has proved that, if $R$ is a right and left noetherian ring with an identity and if the proper prime ideals of $R$ are maximal, then $R$ has a right and left artinian two-sided quotient ring. Robson [5, Theorem 2.11] and Small [6, Theorem 2.13] have proved independently that, if $R$ is a commutative noetherian ring, then $R$ has an artinian quotient ring if and only if the prime ideals of $R$ that belong to the zero ideal are all minimal. We shall generalise these results by proving the

THEOREM. Let $R$ be a right and left noetherian ring with a regular element. Then $R$ has $a$ right and left artinian two-sided quotient ring if and only if each prime ideal of $R$ consisting of zero-divisors is minimal.

1. Small's Theorem. Let $S$ be a non-empty subset of a ring $R$. Then the right annihilator of $S$ in $R$, denoted by $r(S)$, is $\{r \in R: S r=0\}$. The left annihilator, $l(S)$, is $\{r \in R: r S=0\}$. If $c \in R$ we shall denote the right and left annihilators of $\{c\}$ by $r(c)$ and $l(c)$, respectively.

An element $c$ of $R$ is right regular if $r(c)=0$ and is left regular if $l(c)=0$. If $c \in R$ and $l(c)=r(c)=0$, then $c$ is called regular. For any ideal $I$ of $R$ we set

$$
\mathscr{C}^{\prime}(I)=\{r \in R:[r+I] \text { is a right regular element of } R / I\}
$$

and

$$
\mathscr{C}(I)=\{r \in R:[r+I] \text { is a left regular element of } R / I\} .
$$

In addition, we set $\mathscr{C}(I)=\mathscr{C}^{\prime}(I) \cap^{\prime} \mathscr{C}(I)$. In this notation $\mathscr{C}(0)$ denotes the set of regular elements of $R$. If $r \in R$ and $r \notin \mathscr{C}(0)$, then $r$ is called a zero-divisor of $R$.

From now on " ring" will mean " ring with a regular element".

A ring $Q$ with an identity is said to be the right quotient ring of a ring $R$ if

(i) $R \subseteq Q$,

(ii) every regular element of $R$ has an inverse in $Q$, and

(iii) every element of $Q$ has the form $r c^{-1}$ with $r \in R$ and $c \in \mathscr{C}(0)$.

There is an analogous definition of the left quotient ring of $R$. If a ring $R$ has a right quotient ring $Q_{1}$ and a left quotient ring $Q_{2}$, then $Q_{1}=Q_{2}$ and we call $Q_{1}$ the $t$ wo-sided quotient ring of $R$.

Let $M$ be a multiplicatively closed set of elements of a ring $R$. Let $I$ be any ideal of $R$. We shall say that $R$ satisfies the right Ore condition with respect to $M$ modulo $I$ if, for given $r \in R, m \in M$, there exist $r_{1} \in R, m_{1} \in M$ such that $r m_{1}-m r_{1} \in I$. In the special case when $I=0$, we shall say simply that $R$ satisfies the right Ore condition with respect to $M$. There are analogous left-handed definitions. 
THEOREM 1.1. (See [4, p. 118].) A necessary and sufficient condition for a ring $R$ to have $a$ right (left) quotient ring is that $R$ satisfies the right (left) Ore condition with respect to $\mathscr{C}(0)$.

A ring $R$ will be called right artinian if $R$ has an identity and $R$ satisfies the minimum condition for right ideals. There is an analogous definition for left artinian.

The sum of all the nilpotent ideals of a ring $R$ is called the nilpotent radical of $R$. A prime ideal $P$ of $R$ is minimal if $P \supseteq P^{\prime}$, with $P^{\prime}$ a prime ideal of $R$, implies $P=P^{\prime}$. If $R$ is a right noetherian ring (i.e., if $R$ satisfies the maximum condition for right ideals), then the family of proper ideals $\{r(T): T$ is a nonzero ideal of $R\}$ has maximal members and these ideals are called the maximal right annihilators of $R$. There is an analogous definition of the maximal left annihilators of $R$. The next result can easily be checked.

LEMMA 1.2. Let $R$ be a right noetherian ring. Let $P$ be a maximal right annihilator of $R$. Then.

(i) $P$ is a prime ideal of $R$, and

(ii) $P$ consists of zero-divisors of $R$.

We recall the following results.

THEOREM 1.3. (See [3, Theorem 1.5].) Let $R$ be a right noetherian ring and let $N$ be the nilpotent radical of $R$. Then $\mathscr{C}^{\prime}(0) \subseteq \mathscr{C}(N)$. When $r \in R, c \in \mathscr{C}^{\prime}(0)$, there exist $r_{1} \in R, c_{1} \in \mathscr{C}(N)$ with $r c_{1}=c r_{1}$. $\mathscr{C}(N)$.

COROLlaRY 1.4. If $\mathscr{C}^{\prime}(0)=\mathscr{C}(N)$, then $R$ satisfies the right Ore condition with respect to

TheOREM 1.5. (See [6, Theorems 2.11 and 2.12].) Let $R$ be a right noetherian ring with nilpotent radical $N$. Then $R$ has a right artinian right quotient ring if and only if $\mathscr{C}(N) \subseteq \mathscr{C}(0)$.

Theorem 1.6. (See $[3,1.11]$.) Let $R$ be a right noetherian ring with nilpotent radical $N$. Let $P$ be a prime ideal of $R$. Then $P$ is minimal if and only if $P$ does not meet $\mathscr{C}(N)$.

Combining Theorems 1.5 and 1.6, we have

COROLlaRY 1.7. Let $R$ be a right noetherian ring which has a right artinian right quotient ring. Let $P$ be a prime ideal of $R$ consisting of zero-divisors. Then $P$ is a minimal prime ideal.

LEMMA 1.8. Let $R$ be a right and left noetherian ring with nilpotent radical $N$. Suppose that $R$ satisfies the right Ore condition with respect to $\mathscr{C}(N)$. Let $J=\{a \in R: a c=0$ for some $c \in \mathscr{C}(N)\}$. Then

(i) $J$ is an ideal of $R$ and $\mathscr{C}(N) \subseteq \mathscr{C}(J)$,

(ii) there exists $c \in \mathscr{C}(N)$ such that $J c=0$.

Proof. (i) See [2, Chapter 5, Notes on Chapters 3, 4, 5, Propositions 1 and 3].

(ii) Choose $c \in \mathscr{C}(N)$ such that $l(c)$ is maximal in the family of left ideals $\{l(e): e \in \mathscr{C}(N)\}$. If $a \in J$, then there exists $d \in \mathscr{C}(N)$ such that $a d=0$. Since $R$ satisfies the right Ore condition with respect to $\mathscr{C}(N)$, it follows that there exist $d_{1} \in \mathscr{C}(N), c_{1} \in R$ such that $c d_{1}=d c_{1}$. Then $a c d_{1}=0$. By the maximality of $l(c), l(c) \subseteq l\left(c d_{1}\right)$ implies that $l(c)=l\left(c d_{1}\right)$. Therefore $a c=0$. It follows that $J c=0$. 
2. Artinian quotient rings. Throughout this section we shall suppose that $R$ is a right and left noetherian ring (with a regular element).

Lemma 2.1. Let $N$ be the nilpotent radical of $R$. Suppose that $R$ satisfies the right Ore condition with respect to $\mathscr{C}(N)$. Suppose also that each prime ideal of $R$ consisting of zerodivisors is minimal. Then $R$ has a right and left artinian two-sided quotient ring.

Proof. Let $J=\{a \in R: a c=0$ for some $c \in \mathscr{C}(N)\}$. By Lemma 1.8, $J$ is an ideal of $R$ and $J c=0$ for some $c \in \mathscr{C}(N)$. If $J \neq 0$, then $r(J)$ is contained in some maximal right annihilator $P$ of $R$. By Lemma 1.2(i), $P$ is a prime ideal of $R$. Since $P$ meets $\mathscr{C}(N)$, Theorem 1.6 shows that $P$ is not minimal. Hence, by hypothesis, $P$ does not consist of zero-divisors. This contradicts Lemma 1.2(ii). It follows that $J=0$ and, by Lemma 1.8 , that $\mathscr{C}(N) \subseteq \mathscr{C}(0)$. Finally Theorem 1.5 shows that the right and left noetherian ring $R$ has a right and left artinian two-sided quotient ring.

THEOREM 2.2. Let $R$ be a right and left noetherian ring. Then $R$ has a right and left artinian two-sided quotient ring if and only if each prime ideal of $R$ consisting of zero-divisors is minimal.

Proof. The necessity is proved by Corollary 1.7 .

Conversely, suppose that each prime ideal consisting of zero-divisors is minimal. Let $N$ be the nilpotent radical of $R$. By Levitzki's Theorem, $N$ is nilpotent. Therefore there exists a positive integer $s$ such that $N^{s-1} \neq 0, N^{s}=0$.

By Lemma 2.1, to prove that $R$ has a right and left artinian two-sided quotient ring it is sufficient to prove that $R$ satisfies the right Ore condition with respect to $\mathscr{C}(N)$. By Theorem $1.3, R$ satisfies the right Ore condition with respect to $\mathscr{C}(N)$ modulo $N$. Suppose that $1 \leqq k \leqq s-1$ and that $R$ satisfies the right Ore condition with respect to $\mathscr{C}(N)$ modulo $N^{k}$. We shall prove that $R$ satisfies the right Ore condition with respect to $\mathscr{C}(N)$ modulo $N^{k+1}$. The proof will be given in a series of lemmas.

Lemma 2.3. Let $K=\left\{r \in R: r c \in N^{k+1}\right.$ for some $\left.c \in \mathscr{C}(N)\right\}$. Then $K$ is an ideal of $R$.

Proof. Note first that $K \subseteq N$. Let $r_{1}, r_{2} \in K$. Then there exist $c_{1}, c_{2} \in \mathscr{C}(N)$ such that $r_{1} c_{1} \in N^{k+1}, r_{2} c_{2} \in N^{k+1}$. Moreover, there exist $d \in \mathscr{C}(N), a \in R, b \in N^{k}$ such that $c_{1} d-c_{2} a=b$. Therefore

$$
\left(r_{1}-r_{2}\right) c_{1} d=r_{1} c_{1} d-r_{2}\left(c_{2} a+b\right)=\left(r_{1} c_{1}\right) d-\left(r_{2} c_{2}\right) a-r_{2} b \in N^{k+1}
$$

since $r_{2} \in N$. Let $r \in K, x \in R$. Then clearly $x r \in K$. On the other hand, there exists $c \in \mathscr{C}(N)$ such that $r c \in N^{k+1}$. In addition there exist $e_{1} \in \mathscr{C}(N), x_{1} \in R$ such that $x e_{1}-c x_{1} \in N^{k}$. Then $r x e_{1} \in N^{k+1}$. This implies that $r x \in K$. It follows that $K$ is an ideal of $R$.

LEMMA 2.4. $\mathscr{C}(0) \subseteq \mathscr{C}(K)$.

Proof. By Theorem 1.3, $\mathscr{C}(0) \subseteq \mathscr{C}(N)$. Hence $\mathscr{C}(0) \subseteq ' \mathscr{C}(K)$. Suppose that $r \in R$, $c \in \mathscr{C}(0)$ and $c r \in K$. For each positive integer $t$ we set $L_{t}=\left\{r \in R: c^{t} r \in K\right\}$. Since $R$ is right noetherian, the ascending chain of right ideals $L_{1} \subseteq L_{2} \subseteq \ldots$ must terminate. That is, there exists $n$ such that $L_{n}=L_{n+1}$. By Theorem 1.3, there exist $c_{1} \in \mathscr{C}(N), r_{1} \in R$ such that $c^{n} r_{1}=r c_{1}$. 
Now $c r \in K$ implies $c^{n+1} r_{1} \in K$. By the choice of $n, c^{n} r_{1} \in K$ and hence $r c_{1} \in K$. It follows that $r \in K$. In this way, $\mathscr{C}(0) \subseteq \mathscr{C}^{\prime}(K)$. Hence $\mathscr{C}(0) \subseteq \mathscr{C}(K)$.

Lemma 2.5. Let $T=\{r \in R: c r \in K$ for some $c \in \mathscr{C}(N)\}$. Then $T$ is an ideal of $R$ and there exists $c \in \mathscr{C}(N)$ such that $c T \subseteq K$.

Proof. Since $K \subseteq N$, the ideal $N / K$ is the nilpotent radical of the ring $R / K$. In addition, it is clear that $\mathscr{C}(N) \subseteq^{\prime} \mathscr{C}(K)$. By Theorem $1.3,{ }^{\prime} \mathscr{C}(K)=\mathscr{C}(N)$. Then Corollary 1.4 shows that $R / K$ satisfies the left Ore condition with respect to $\mathscr{C}(N / K)$. The result now follows by Lemma 1.8.

Note. The proof of Lemma 2.5 uses the left hand versions of Theorem 1.3 and Lemma 1.4.

LEMMA 2.6. $T=K$.

Proof. Note first that $K \subseteq T$. Let $V=\{r \in R: r T \subseteq K\}$. Then $V$ is an ideal of $R$ and $K \subseteq V$. If $T \neq K$, then $V / K$ is contained in a maximal left annihilator $P^{\prime}$ of $R / K$. By Lemma 1.2(i), $P^{\prime}$ is a prime ideal of $R / K$. Therefore $P^{\prime}=P / K$ for some prime ideal $P$ of $R$ with $K \subseteq V \subseteq P$. By Lemma 2.5, $P$ meets $\mathscr{C}(N)$ and, by Theorem 1.6, $P$ is not minimal. By hypothesis, $P$ meets $\mathscr{C}(0)$. Hence, by Lemma $2.4, P$ meets $\mathscr{C}(K)$. That is, $P^{\prime}$ does not consist of zero-divisors of $R / K$. This contradicts Lemma 1.2(ii). Hence $T=K$.

COROLLARY 2.7. $\mathscr{C}(N) \subseteq \mathscr{C}(K)$.

Proof. Let $r \in R, c \in \mathscr{C}(N)$. If $r c \in K$, then clearly $r \in K$. On the other hand, if $c r \in K$, then $r \in T=K$.

LEMMA 2.8. $R$ satisfies the right Ore condition with respect to $\mathscr{C}(N)$ modulo $N^{k+1}$.

Proof. Recall that $N / K$ is the nilpotent radical of $R / K$. By Theorem 1.3 and Corollary $2.7, \mathscr{C}(N)=\mathscr{C}(K)$. Then Theorem 1.3 also shows that $R / K$ satisfies the right Ore condition with respect to $\mathscr{C}(N / K)$. In other words, $R$ satisfies the right Ore condition with respect to $\mathscr{C}(N)$ modulo $K$. It follows easily that $R$ satisfies the right Ore condition with respect to $\mathscr{C}(N)$ modulo $N^{k+1}$.

We recall that $N^{s}=0$. Therefore, by induction, $R$ satisfies the right Ore condition with respect to $\mathscr{C}(N)$. As we remarked earlier, this allows us to conclude that $R$ has a right and left artinian two-sided quotient ring. This completes the proof of Theorem 2.2.

It might be conjectured that, if $R$ satisfies the condition that

$$
\text { each prime ideal which does not meet } \mathscr{C}^{\prime}(0) \text { is minimal, }
$$

then $R$ has a right and left artinian two-sided quotient ring. The following example of Small [7] shows that this conjecture is false.

Example. Let $Z$ denote the ring of integers. Let $p$ be a prime in $Z$. Let $S$ be the ring of all two-by-two " matrices" of the form

$$
\left(\begin{array}{ll}
a & 0 \\
b & c
\end{array}\right)
$$


with $a \in Z, b \in Z /(p)$ and $c \in Z /(p)$. Addition in $S$ is defined component-wise and multiplication is given by

$$
\left(\begin{array}{ll}
a & 0 \\
b & c
\end{array}\right)\left(\begin{array}{ll}
a^{\prime} & 0 \\
b^{\prime} & c^{\prime}
\end{array}\right)=\left(\begin{array}{cc}
a a^{\prime} & 0 \\
b a^{\prime}+c b^{\prime} & c c^{\prime}
\end{array}\right)
$$

where $Z$ acts on $Z /(p)$ in the usual way. Then $S$ has the following properties (see [7]).

(i) $S$ is a right and left noetherian ring with an identity.

(ii) If $N$ is the nilpotent radical of $S$, then

$$
N=\left\{\left(\begin{array}{ll}
0 & 0 \\
b & 0
\end{array}\right): b \in Z /(p)\right\} \text {. }
$$

(iii) $S$ has a two-sided quotient ring $Q$ but $Q$ is neither right nor left artinian.

It is not hard to prove that $S$ has the further property:

(iv) $\mathscr{C}(N)=\mathscr{C}^{\prime}(0)=\left\{\left(\begin{array}{ll}a & 0 \\ b & c\end{array}\right): a \neq 0\right.$ and $\left.c \neq 0\right\}$.

Combining (iv) with Theorem 1.6 we have immediately:

(v) $S$ satisfies (*).

Finally, let

$$
r=\left(\begin{array}{ll}
0 & 0 \\
1 & 0
\end{array}\right) \in S \text { and } c=\left(\begin{array}{ll}
p & 0 \\
0 & 1
\end{array}\right) \in \mathscr{C}(N)
$$

Then there does not exist $c^{\prime} \in \mathscr{C}(N)$ such that $c^{\prime} r \in S c$. Using this fact it is not hard to prove that

(vi) $S$ satisfies the right Ore condition with respect to $\mathscr{C}(N)$ but $S$ does not satisfy the left Ore condition with respect to $\mathscr{C}(N)$.

\section{REFERENCES}

1. M. Djabali, Anneaux de fractions généralisés artiniens, C. R. Acad. Sci. Paris 268 (1969), 2138-2140. 1961.

2. A. W. Goldie, Rings with maximum condition, Mimeographed lecture notes, Yale University,

3. A. W. Goldie, Lectures on non-commutative noetherian rings, Canad. Math. Congress, York University, Toronto, 1967.

4. N. Jacobson, The theory of rings, Amer. Math. Soc. Surveys No. 2 (New York, 1943).

5. J. C. Robson, Artinian quotient rings, Proc. London Math. Soc. (3) 17 (1967), 600-616.

6. L. W. Small, Orders in artinian rings, J. Algebra 4 (1966), 13-41.

7. L. W. Small, On some questions in noetherian rings, Bull. Amer. Math. Soc. 72 (1966), 853-857.

\section{UNIVERSITY OF GLASGOW}

GLASGOW, G12 8QQ 